\title{
La détermination passive et quelques thématisations particulières en anglais médical
}

Didier Carnet

\section{OpenEdition}

\section{Journals}

Édition électronique

URL : http://journals.openedition.org/asp/3373

DOI : 10.4000/asp.3373

ISSN : 2108-6354

\section{Éditeur}

Groupe d'étude et de recherche en anglais de spécialité

\section{Édition imprimée}

Date de publication : 1 décembre 1997

Pagination : 505-524

ISSN : 1246-8185

\section{Référence électronique}

Didier Carnet, «La détermination passive et quelques thématisations particulières en anglais médical », ASp [En ligne], 15-18 | 1997, mis en ligne le 05 mars 2013, consulté le 01 mai 2019. URL : http:// journals.openedition.org/asp/3373 ; DOI : 10.4000/asp.3373

Ce document a été généré automatiquement le 1 mai 2019.

Tous droits réservés 


\title{
La détermination passive et quelques thématisations particulières en anglais médical
}

\author{
Didier Carnet
}

\section{Introduction}

1 L'anglais médical peut se définir comme l'usage de l'anglais pour rendre compte de connaissances médicales : c'est de l'anglais et en même temps, c'est le vecteur de savoirs et de savoir-faire. Il n'y a bien évidemment pas de grammaire linguistique spécifique, mais une utilisation spécifique et parfois massive de certains points particuliers. Ceci permet de mettre en place une stratégie énonciative propre que je vais tenter de cerner et de définir, à travers ce que j'ai appelé la détermination passive et quelques thématisations particulières. En effet, l'usage du passif - et c'est un fait bien connu - est beaucoup plus répandu chez les médecins, voire les scientifiques en général, qu'en anglais littéraire et standard. Les scientifiques eux-mêmes, conscients de cette utilisation massive de la diathèse passive, ont tenté d'en limiter l'usage. En effet, dans un fascicule publié en 1961 pour aider les médecins dans la rédaction de leurs articles, The Handbook for Chemical Society Authors, il est recommandé d'éviter une trop grande utilisation de cette diathèse passive :

Before the final typing every paper should be scrutinised to see whether it cannot be improved by eliminating abstract words and passive voices.

2 Il est cependant amusant de noter le poids de la tradition et des habitudes. Cette recommandation comporte elle-même deux verbes construits à la diathèse passive ! Elle est proche de l'axiome "Faites ce que je dis, mais non ce que je fais». Il aurait effectivement été possible de dire : «Before the final typing you should scrutinise every paper to see whether you cannot improve it by eliminating abstract words and passive voices ». 
Donc le «problème » de la diathèse passive existe bien. Il est posé et je vais tenter de le résoudre. $\mathrm{Y}$ a-t-il vraiment adéquation entre la recommandation initiale et ce que je propose ? La diathèse passive correspond-elle à un besoin stylistique et rhétorique des scientifiques? A-t-elle toujours la même valeur, le même rôle, la même fonction? Je vais tenter d'y répondre en analysant les multiples exemples de mon corpus médical.

\section{Corpus}

4 J'ai travaillé à partir d'un corpus suffisamment vaste pour obtenir un échantillon représentatif de ces caractéristiques médicales. J'ai utilisé la revue spécialisée anglaise The Lancet qui est publiée depuis 1823 et que j'ai dépouillée depuis 1940. J'ai analysé de façon systématique les numéros parus de 1990 à 1995 et j'ai alors sélectionné un corpus comprenant une centaine d'articles médicaux, me permettant de comparer 2115 structures passives. Je n'ai pas utilisé uniquement les articles de recherche à la forme canonique de type IMRAD : Introduction, Materials and Methods, Results and Discussion. J'ai au contraire volontairement utilisé tous les types d'articles publiés hebdomadairement dans The Lancet, dont les rubriques les plus régulières sont Editorial, Commentary, Articles, Short reports, Public health, Clinical practice, Viewpoint, Bookshelf, News, Letter to the editor. J'ai ainsi voulu obtenir un échantillon le plus représentatif possible de The Lancet afin de réaliser une étude linguistique de cette revue. D'autre part, je n'ai pris en compte que le texte définitif publié, sans prendre en considération les corrections éventuelles des reviewers. Je considère le "produit fini », publié en tant que représentation du mode de pensée des médecins. Ainsi le terme d'«énonciateur", qui désigne celui qui prend en charge l'énoncé, renverra-t-il dans le cadre de cette étude au médecin-écrivain, le médecin au sens large, la communauté de médecins publiant dans The Lancet.

\section{Définition traditionnelle}

Que ce soit en français ou en anglais, la description de la diathèse passive a toujours été faite traditionnellement par rapport à la diathèse active. À titre d'exemple, nous citerons la Grammaire Larousse du français contemporain qui se contente d'énoncer une règle excessivement simple, voire simpliste :

RÈGLE. Toute forme verbale qui admet une construction transitive directe peut, par retournement, devenir passive. [...] Il est possible de changer l'ordre des mots dans les phrases (à condition de modifier la forme verbale) sans que l'idée exprimée varie. (Chevalier et alii $1964: 322$ )

Pour les auteurs, le passif n'est donc qu'un « retournement» de l'actif et les contenus sémantiques sont les mêmes. Le passif est donc traité dans la tradition grammaticale classique essentiellement comme un problème de morphologie verbale. Face à cette présentation du passif, nous sommes alors en droit de nous demander à quoi peut bien servir cette transformation si celle-ci doit aboutir à des phrases identiques.

\section{La théorie de l'énonciation}

7 Une phrase n'est qu'une suite grammaticale que l'on peut transformer à volonté, mais ce qui, hors contexte, n'a pas grand intérêt. En revanche, un énoncé est produit et pris en 
charge par un énonciateur dans une situation particulière. Il peut ainsi moduler son degré de certitude et son degré d'engagement. Il marquera par toute une série de repérages et de déterminations son point de vue. Ces déterminations portent bien sûr à la fois sur le groupe verbal et sur le groupe nominal. Celle-ci est certes réalisée par l'emploi des temps, des aspects et des modalités, mais également par l'utilisation de la diathèse (active ou passive).

8 J'ai donc choisi principalement de travailler sur des objets de discours (médical) et non sur des entités extralinguistiques. C'est l'énonciateur qui choisit, grâce à certains procédés linguistiques, un ordre de présentation des éléments afin de délivrer une information particulière au co-énonciateur. Le contexte est donc primordial, et comme je vais le montrer maintenant, "tout changement syntaxique entraîne un changement sémantique ». (Culioli et alii $1970: 7$ )

9 Le lexique repose sur le concept de notions, c'est-à-dire de termes ayant des propriétés «physico-culturelles » avec des représentations mentales. Ces notions sont antérieures à la catégorisation en nom, verbe... et la relation primitive va établir une relation ordonnée entre ces notions, relations que tout le monde pose de façon plus ou moins implicite. Elle sera appelée lexis et permettra d'orienter cette relation d'une source à un but.

Source et but déterminent une relation primitive et par là un ordre [...] Ordre n'est pas pris au sens d'ordre partiel ou ordre total, mais au sens où lorsqu'il y a un «mangeur » et "un mangé », il y a un ordre entre les deux termes. (Culioli 1975-1976 : 49)

10 Ceci revient à dire que lorsque l'on prend les termes «apple ", « man », " eat », la relation primitive sera : <man eat apple>.

11 À cette relation primitive au niveau profond des notions va correspondre en surface la relation prédicative. Mais alors que la relation primitive est obligatoirement orientée, la relation prédicative, elle, va pouvoir se construire aussi bien à partir de l'élément source que de l'élément but de la relation primitive. Il va y avoir une organisation des éléments "source, relateur, but", et par là même, une orientation de la lexis. Il y a donc orientation de cette relation par la sélection du premier argument, c'est-à-dire du complément de rang zéro. Si C0 correspond à l'élément source de la relation primitive, nous obtiendrons une relation prédicative à l'actif. En revanche, si C0 correspond à l'élément but, nous aurons une relation prédicative au passif.

12 La relation énonciative, elle, va être la prise en compte de cette relation prédicative par un énonciateur. Il va donc y avoir construction de l'énoncé, avec l'élaboration de repères et de déterminations. Ceux-ci seront le choix d'un temps, d'un aspect, d'une modalité, d'une détermination... Mais le tout premier choix, la toute première détermination portera sur le terme de départ de la relation énonciative. Le premier argument sera-t-il le terme source de la relation primitive, utilisant ainsi la relation prédicative à l'actif et créant par là même un "énoncé actif», ou alors le terme but pour obtenir un «énoncé passif »?

13 J'ai classé les exemples issus de mon corpus en trois grandes catégories. Les deux premières, à savoir la détermination passive simple et la détermination passive complexe, ne s'opposent que par le fait que la classe d'agents peut ou non être instanciée. D'autre part, j'ai également pris en compte ce que j'ai appelé « détermination passive tronquée ", mais que l'on aurait pu également qualifier de "passif elliptique » ou encore de "passif synthétique ». Elle correspond à différents cas de figure ayant tous en commun 
l'effacement d'au moins un des termes appartenant à la construction d'une détermination passive.

14 Je présente tout d'abord les résultats bruts de cette étude statistique sous forme de tableau récapitulatif (voir Tableau 1). Le commentaire qui suivra apportera quelques précisions.

\section{Tableau 1}

\begin{tabular}{|c|c|c|c|c|}
\hline & passif d'action & & 827 & $39 \%$ \\
\hline $\begin{array}{l}1 . \\
\text { Détermination }\end{array}$ & passif cataphorique & & 15 & $1 \%$ \\
\hline passive & passif & be $(60)$ & & \\
\hline \multirow[t]{6}{*}{ simple } & statif & remain (6) & 71 & $3 \%$ \\
\hline & & become (5) & & \\
\hline & pseudo-passif & & 11 & $0,5 \%$ \\
\hline & & by & 152 & \\
\hline & & with & 16 & \\
\hline & & by « spécial » & 9 & \\
\hline 2. & & from & 6 & $9 \%$ \\
\hline Détermination & passif d'action & through & 1 & \\
\hline passive & & via & 1 & \\
\hline \multirow[t]{7}{*}{ complexe } & & in & 5 & \\
\hline & & to & 2 & \\
\hline & semi-passif \& & by & 9 & \\
\hline & passif réciproque & to & 21 & $2,5 \%$ \\
\hline & & with & 22 & \\
\hline & & $\begin{array}{l}\text { sans be } \\
\text { sans 2è repérage }\end{array}$ & 204 & $9,5 \%$ \\
\hline & & $\begin{array}{l}\text { sans be } \\
\text { avec 2è repérage }\end{array}$ & 99 & $4,5 \%$ \\
\hline
\end{tabular}




\begin{tabular}{|l|l|l|l|l|}
\hline $\begin{array}{l}\text { Détermination } \\
\text { passive } \\
\text { tronquée }\end{array}$ & passif d'action & $\begin{array}{l}\text { sans be } \\
\text { sans 2è repérage } \\
\text { sans sujet }\end{array}$ & 27 & $1,5 \%$ \\
\hline & & nominalisation & 15 & $1 \%$ \\
\hline & & adjectif composé & 111 & $5 \%$ \\
\hline & passif statif & rôle adjectival & 487 & $23 \%$ \\
\hline & & rôle nominal & 4 & $0,5 \%$ \\
\hline
\end{tabular}

Avant toute explication, une remarque s'impose d'elle-même: la détermination passive sous-entend une classe d'agents, mais celle-ci n'est instanciée que dans $16 \%$ des cas (11,5\% pour la détermination passive complexe et $4,5 \%$ pour la détermination passive tronquée). Le schéma de base de la détermination passive est donc un schéma binaire. Il ne présente que le but de la relation primitive et un processus stabilisé marqué par le participe passé, tous deux mis en relation par une opération d'identification réalisée par «be ». Ayant choisi un « thème » à son discours - le C0 de l'énoncé à la diathèse passive l'énonciateur crée une relation binaire avec classe d'agents à vide. Il peut choisir d'instancier cette classe d'agents en le mentionnant pour apporter des précisions, mettre en relief un événement nouveau, opérer une sélection dans un sous-ensemble, faire une opération de qualification. Mais dans tous les cas, il s'agit là d'une deuxième opération de détermination ne faisant pas partie du schéma de base de la diathèse passive. Ces chiffres renforcent donc ceux avancés par R. Quirk et alii qui nous signalent que l'agent est exprimé approximativement dans un cas sur cinq (1972: 807) et ceux de J. Albrespit qui n'a trouvé que 81 formes passives sur les 682 qu'il a analysées comportaient un complément d'agent, soit $12 \%$ (1994: 378). Dans mon corpus, $84 \%$ des cas relevés présentent donc une classe d'agents à vide, ce qui confirme bien que le passif n'est pas l'inverse de l'actif, mais bien une détermination originale et indépendante très utilisée en anglais médical. Je vais maintenant commenter ce tableau en m'appuyant sur quelques exemples caractéristiques.

\section{La détermination passive simple}

\subsection{Le passif d'action}

Le participe passé renvoie à une agentivité ou à une propriété. Dans ce cas, ses valeurs sont proches de celles de l'adjectif lorsque la propriété est prédiquée hors agentivité. Cette valeur stative est incompatible avec le repérage par rapport à un agent. Il faut qu'il y ait passage d'un état à un autre, donc un point d'instabilité, pour justifier la construction d'une classe d'agents, même si elle est vide, ce qui est donc le cas dans $39 \%$ des énoncés de mon corpus. Voici un exemple caractéristique :

In response to this concern, families are now offered brief educational sessions or workshops to help them understand the illness. (The Lancet vol. 340, Oct. 24, 1992 : 1007) 
17 Le passage d'un état à un autre est ici exprimé par l'adverbe now qui implicitement fait référence à un état antérieur. Il y a donc bien eu changement d'état, et nous distinguons là un passif d'action. La diathèse passive joue un rôle bien spécifique en plaçant l'élément but au centre du discours. La relation primitive est ici <doctor - offer educational sessions family>.

Dans ce cas, un triple choix s'offre à l'énonciateur. Le centre du discours, c'est-à-dire le $\mathrm{C} 0$, pourrait être doctors et l'on aurait alors une diathèse active. Au contraire, l'énonciateur peut choisir entre families et educational sessions, les deux éléments buts de cette relation primitive, car offer (tout comme give ou send) fonctionne avec ce que l'on appelle traditionnellement deux compléments d'objet, direct et indirect. Le choix de l'énonciateur se porte sur families qui représente un animé humain comme centre du discours. Ce choix n'est à nouveau pas le fruit du hasard. Ce terme de départ a déjà subi un repérage préalable dans le texte. Ce sont les familles dont certains membres ont des troubles schizophréniques. Il est en effet impossible de prendre n'importe quel terme comme point de départ de la relation prédicative. Cet argument qui va instancier la place de terme de départ de la relation prédicative doit être déterminé, c'est-à-dire repéré par rapport à la situation d'énonciation. Il y a eu dans ce cas fléchage contextuel sur ce terme. Il devient donc centre du propos, ce qui permet d'une part d'introduire pour la première fois le terme educational sessions et, d'autre part, de présenter la classe d'agents à vide, de ne pas l'instancier, c'est-à-dire de ne pas nommer l'agent doctors.

\section{Prédétermination}

Cet effacement de l'agent doctors est d'ailleurs un procédé récurrent en anglais médical. Dans un article intitulé "Transfusion-associated graft-versus-host disease in immunocompetent patients : a self-protective mechanism » (The Lancet, vol 343 : March 26, 1994), j'ai relevé cinquante-deux occurrences de détermination passive en soixanteneuf lignes, dans la partie intitulée Patients and methods, ce qui représente une très grande fréquence d'utilisation. Sur ces cinquante-deux exemples, aucun ne présente l'agent! Seuls six exemples présentent une deuxième détermination servant à introduire le moyen par lequel telle relation a été réalisée. Dans tous ces exemples, l'agent est complètement effacé. La classe est construite à vide, car le préconstruit est bien qu'il y ait une origine au procès, mais cet élément est tellement évident qu'on ne l'identifie plus. Il s'agit des coauteurs de l'article qui ont été présentés tout au début. Les relations primitives sont bien sûr, we, doctors, tested, obtained, studied, screened, re-stimulated, separated... Nous assistons donc à une in-détermination, ou plutôt une non-détermination qui correspond à un préconstruit, ou mieux encore, à une prédétermination au niveau des relations primitives. L'agent étant connu grâce à ce préconstruit, ou prédétermination, il ne sera plus mentionné, ce qui permet d'éviter toute une série de répétitions qui seraient fastidieuses, et tout à fait inutiles. Et lorsqu'une nouvelle opération de détermination a lieu, elle sert non pas à présenter l'agent, mais le moyen ou la manière, à l'aide de divers relateurs tels que with et by. Cette prédétermination ou détermination implicite est en quelque sorte l'un des principes de base de l'utilisation de la détermination passive en anglais médical.

\section{Objectivité}

Si la détermination passive simple correspond principalement à une prédétermination implicite de l'élément source, elle prend également souvent une valeur de sens particulière. En effet, l'énonciateur efface l'agent par un style impersonnel et objectif, et 
tente d'atteindre une description et une vérité quasi universelle. Cela se retrouve principalement dans les conclusions d'articles médicaux où le médecin vise à obtenir une objectivité parfaite. Voici l'un des exemples de mon corpus :

Over 50 years later the test has again been shown to be of limited value in heart failure. Surely the ESR should no longer be included in the standard investigations for heart failure, except perhaps in those in whom endocarditis is suspected. Research endeavours might likewise be more usefully deployed elsewhere. (The Lancet vol. 337, April 20, 1991 : 949).

Il est intéressant de noter que cette conclusion n'utilise que des déterminations passives. De plus, nous pouvons relever la présence d'une très forte modalité de type épistémique. Nous avons deux auxiliaires modaux should et might à valeur épistémique, tous deux étant renforcés par des adverbes ayant la même valeur surely et perhaps. Ce type d'énoncé passif à modalité épistémique est presque toujours construit sans deuxième détermination, c'est-à-dire sans agent. Leur rôle consiste justement à ne pas poser de limites à la classe des agents, à construire une classe la plus large possible. En effet, chaque fois qu'un agent est mentionné se crée un effet de restriction, de précision ou de renseignement supplémentaire. Cette détermination passive de type épistémique permet à l'énonciateur à la fois de ne pas trop s'engager, puisque le procès est instanciable dans toute situation, quel que soit l'agent, et d'atteindre une sorte d'universalité et d'objectivité totales, puisqu'il s'efface en tant qu'agent particulier. La prédication peut être effectuée par tout agent, tout médecin! Il ne pose aucune limite à cette classe qui est alors une classe ouverte. Prédétermination et objectivité sont donc deux causes primordiales qui expliquent que la détermination passive soit si fréquente en anglais médical. Elles permettent à la fois des énoncés impersonnels et des descriptions précises centrées sur le thème de l'étude, plus que sur celui qui étudie.

\subsection{La détermination passive cataphorique}

Nous avons dans ce cas le schéma de base d'une détermination passive simple, à savoir C0 + be + participe passé, la classe d'agents étant construite à vide :

Since the main determinant of the sedimentation rate seems to be the plasma fibrinogen content, and in heart failure there are reports of increased concentration of fibrinogen, it might have been supposed that the ESR would increase when the heart fails. (The Lancet vol. 337, April 20, 1991 : 949).

La particularité de cet exemple vient du fait que Co est représenté par le pronom it, et que ce pronom n'est pas anaphorique, mais cataphorique. En effet, il ne reprend pas un terme qui est déjà présent précédemment, mais au contraire, il s'agit d'une opération de renvoi à un terme qui suit immédiatement. En fait, it représente (the fact) that the ESR would increase when the heart fails. Il s'agit donc plus encore qu'un simple renvoi cataphorique à un terme, car it annonce un ensemble complexe constitué d'une proposition principale et d'une proposition subordonnée. En résumé, disons que it renvoie à la relation prédicative $<$ the ESR - increase>. Cette construction particulière correspond donc à une contrainte syntaxique. Nous la retrouvons systématiquement lorsque C0 est trop long et développé. Il est alors remplacé par it + be + participe passé + le véritable sujet grammatical. D'autre part, cette construction est liée à la présence de verbes ayant une forte valeur modale. Il faut enfin noter que si cette construction apparait pour déplacer et repousser en fin de phrase un Co qui serait trop lourd s'il se trouvait à sa place habituelle, elle correspond également à une fonction bien précise. Cette détermination passive cataphorique permet 
une focalisation différente sur un élément. Il y a mise en valeur, sans que cet élément soit placé en tête de phrase. Ce type de détermination passive est singulier en ce sens qu'il y a une double opération. Tout d'abord thématisation de it placé en début d'énoncé, puis focalisation particulière, par le renvoi cataphorique, sur tout ce qui suit le participe passé. Il y a donc, en quelque sorte, une double focalisation qui permet de ne pas instancier la classe d'agents. Aucune restriction n'est envisagée sur cette classe d'agents, et cela confère à l'énoncé une valeur quasi générale. L'agent du procès est inconnu ou indéterminé, mais ce qui compte, c'est l'effet de double focalisation qui permet de souligner le thème du discours.

\subsection{Le passif statif : rôle adjectival}

En règle générale, le participe passé peut renvoyer à une agentivité ou à une propriété. Lorsque la propriété est prédiquée hors agentivité, les valeurs sont proches de celles de l'adjectif. C'est ce que nous obtenons dans certains exemples caractéristiques de notre corpus :

In some women, asthma symptoms improved and they needed less therapy, but again the picture was mixed. (...)These high levels inhibit the formation of gap junctions (pathways of low electrical resistance) in the smooth muscle of the uterus, which remains largely relaxed and poorly contractile. (The Lancet vol. 335, Jan. 27,1990 : 204).

Most of the cell activities discussed thus far maintain the life of the cell on a day-today basis. However, cells become damaged, diseased or worn out - and die. (Tortora $1992: 47)$.

Il est à noter que dans ce cas, remain, become (et get) peuvent être utilisés à la place de l'auxiliaire be. Nous avons alors une valeur purement adjectivale indiquant que l'on est dans du statif, qu'une coupure est opérée entre l'origine de l'événement et le moment de l'énonciation. Il n'y a pas d'intervention d'un agent au moment d'énonciation, même si cette intervention a forcément eu lieu à un moment donné. Les choses sont présentées sous forme de résultat statif, et donc l'agentivité passe au second plan. Il va donc de soi que la place de l'agent n'est pas instanciée et est construite à vide. L'agent n'a alors plus aucune importance ni même raison d'être. Dans ces cas, le participe passé renvoie bien sûr à l'accompli. Il représente un processus stabilisé, c'est-à-dire un état. Son utilisation est quasiment adjectivale, et nous pouvons le considérer comme attribut. Nous verrons plus loin qu'il peut également être utilisé comme épithète, dans le cas de détermination passive tronquée. Mais nous ne devons pas considérer ce participe passé comme un adjectif à part entière. La marque -ed est une flexion de nature grammaticale et non pas un affixe de nature lexicale. Autrement dit, cette flexion donne une autre forme à une même lexie, alors que l'affixe forme, lui, une nouvelle lexie. Nous ne pourrons donc pas regarder un participe passé comme un véritable adjectif. Il n'y a pas véritablement conversion. Cependant, nous parlerons, dans le cas de passif statif, de fonctionnement adjectival.

\subsection{Le pseudo passif}

Nous reprenons cette appellation de F.R. Palmer (1965: 87), bien que cette catégorie soit très proche de celle regroupant les passifs statifs. Voici l'un des exemples de ce type : 
Teaching districts also have more consultants and for historical reasons tend to be situated in deprived inner city areas with higher suicide rates. (The Lancet vol. 344, Sept. 17, $1994: 822$ ) dans la première opération de détermination, va permettre d'effectuer une opération de repérage par rapport au terme choisi, et d'exclure tous les autres agents possibles. Nous sommes donc en présence de deux opérations de repérage successives, la première servant à déterminer l'élément but de la relation primitive, et la seconde l'élément source. Ces deux opérations sont bien des opérations de détermination puisque la construction consiste, par une série de relations sur des relations, à introduire au fur et à mesure des délimitations. Cette opération de repérage supplémentaire va donc apporter plus de détermination. Elle va lever une certaine indétermination inhérente au schéma de base du passif; et ce, à l'aide de divers marqueurs. En effet, l'opération de repérage de la détermination passive simple était réalisée le plus souvent par le marqueur be. Mais nous avons montré que d'autres relateurs peuvent être utilisés tels que remain et become (et get ). De la même manière, différents relateurs vont être utilisés pour réaliser cette deuxième opération de repérage, qui va, en quelque sorte, apporter une "surdétermination passive ». C'est ainsi qu'en parallèle à by qui sert à introduire l'agent, le terme qui contrôle l'événement, nous avons relevé les relateurs suivants :

With exprime le moyen, la manière, by « spécial » exprime également le moyen, from sert à particulariser l'agent, via exprime le moyen (by way of, by means of), through l'intermédiaire permettant à l'agent d'accomplir le changement d'état, in ainsi que to une opération de localisation. Voici un exemple caractéristique pour chacun de ces marqueurs :

The concept of expressed emotion was introduced over thirty years ago by Brown and his colleagues. (The Lancet, vol. 344, Oct. 24, 1992 : 1007)

Prior developmental assessments had been carried out with 67 (76\%) and 40 (45\%) of the dropouts at 5 and 20 months, respectively, by the Griffiths test. (The Lancet vol. 345, Feb. 18, 1995 : 447)

The data were obtained from the Family Health Study of the New York State Institute for Basic Research in Development Disabilities. (The Lancet vol .344, Oct. 15, 1994 : 1093)

The large majority of lung cancers are caused by carcinogens and tumour promoters which are ingested via cigarette smoking. (The Lancet vol 335, Feb. 3, $1990: 262$ ).

Most effects are mediated through binding to sex-hormone-binding globulin (SHBG) or to androgen receptors. (The Lancet vol. 340, Nov. 21, $1992: 1264$ ) 
[...] the drug is relatively ineffective against E. histolytica in the colon, because the drug is so completely absorbed in the small intestine. (The Lancet vol. 343, May 28, $1994: 1352$ )

The effect of a very low dose does not depend on homeopathy: even at an international normalised ratio (INR) of 1-5 clotting factor concentrations are reduced to 50-70\% of normal. (The Lancet vol. 343, April 9, $1994: 867$ ) complexe en ce sens qu'elle présente une deuxième opération de détermination, mais également parce que différents marqueurs peuvent être utilisés afin de présenter diverses opérations de repérage.

\section{Thématisation}

31

J'ai donc constaté que la première opération de repérage dans la démarche de constitution d'un énoncé consiste à assigner à l'intérieur de la relation prédicative une valeur terme de départ à l'un des éléments de la relation primitive. Ce terme de départ est, du point de vue de la relation énonciative, ce que l'on peut appeler le thème. C'est en cela que l'on peut dire que la détermination passive correspond à une opération de thématisation. Nous reprenons là l'opposition «thème/rhème » qui appartient à la terminologie praguoise classique. En linguistique de langue anglaise, il conviendrait d'utiliser les termes « topic/comment » et de parler de «topicalization ». Cette thématisation entraîne obligatoirement une focalisation particulière sur le groupe nominal sujet de la relation prédicative. En fait, la détermination passive attire moins l'attention sur le verbe que sur l'élément qui est choisi comme sujet grammatical. C'est un problème de détermination de ce groupe nominal sujet, lié en réalité à la question de repère constitutif de l'énoncé. N'oublions pas que ce sont des problèmes de relation portant sur des valeurs qui ne sont pas des valeurs de termes isolés, mais de termes qui appartiennent à une relation! Ce procédé de thématisation consiste donc en une mise en valeur d'un élément réalisée en le plaçant en tête de phrase. Il y a donc forcément focalisation sur cet élément (la réciproque n'étant pas forcément vraie: il peut très bien $\mathrm{y}$ avoir focalisation contrastive, intonative - sur un élément sans qu'il y ait pour autant thématisation!).

Voici un exemple issu d'un texte dont le titre est «Expressed emotion in schizophrenia ». Il est évident d'après ce titre que le thème de l'article sera l'extraversion. Cependant, l'auteur décide dès la première phrase d'insister à nouveau sur son propos et sur le thème de son discours.

The concept of expressed emotion was introduced over 30 years ago by Brown and his colleagues to explain why some schizophrenic patients relapsed despite adequate drug therapy. (The Lancet vol. 340, Oct. 24, 1992 : 1007)

L'agent responsable de ce concept, de même que la date d'apparition du concept n'apparaissent qu'en commentaire du thème principal. L'intérêt de l'auteur n'est pas historique. Il ne veut par là n'apporter qu'une précision supplémentaire, servant à justifier l'intérêt qu'il porte à ce concept qui est bien le centre de son propos, le thème de son discours. En revanche, la démarche est tout à fait différente avec ce deuxième exemple.

In 1936, Paul Wood described 22 patients with congestive heart failure in whom the ESR declined as failure worsened and rose as the condition improved. (...) Mc Ginnis et al found that in many cases the ESR was raised and remained high after treatment. Sanghvi found that the ESR was normal in $50 \%$ of patients with cardiac failure from several underlying causes. (The Lancet vol. 337, April 20, 1991 : 949) 
article est intitulé "Not the ESR again ». L'auteur va donner son avis sur le rôle de la vitesse de sédimentation dans le cas de crises cardiaques. Mais pour ce faire, il va dresser l'historique des différents points de vue au cours du siècle. Cette fois-ci, le thème de ses différents énoncés ne sera plus la vitesse de sédimentation, mais les médecins qui ont fait des recherches scientifiques. Il est clair que l'orientation de la relation prédicative n'est pas due au hasard. Plusieurs études sont rapidement décrites et leurs auteurs sont présentés comme les thèmes des différentes relations prédicatives. Tout cela fait sens et correspond à une véritable stratégie énonciative. Le premier auteur ne voulait qu'apporter un complément d'information secondaire, il place l'élément source en deuxième position, alors que le second auteur veut insister sur les contradictions des études précédant la sienne, il place les éléments sources en première position. Ces deux exemples corroborent bien la thèse que le passif n'est pas un simple « retournement » de l'actif comme nous l'affirmions déjà au début de cette étude. Toute présentation de surface par rapport aux relations primitives ordonnées est significative. Toute modification par rapport à l'ordre des relations primitives a un sens et n'est pas le fruit du hasard.

ans certains cas, l'énonciateur peut choisir de thématiser le prédicat en le gardant sous sa forme de participe passé. Il y aura alors focalisation et intention particulières de la part de l'énonciateur :

Also brought up in the debate was the concept of fuel teratogenesis, according to which the effects of poor glycaemic control on foetal development are time dependent and cumulative during gestation. (The Lancet vol. 345, April 8, 1995 : 915)

Il y a là focalisation et intention particulières de la part de l'énonciateur. Il aurait pu dire : "The concept of fuel teratogenesis was also brought up in the debate. According to it... ». Dans ce cas, nous aurions eu une détermination passive habituelle avec thématisation de l'élément but de la relation primitive. Mais ici, l'énonciateur choisit de commencer par le participe passé, c'est-à-dire le prédicat et de ne présenter Co que plus loin dans l'énoncé, créant ainsi une inversion sujet - prédicat. Il y a donc focalisation sur ce prédicat. Ce qui compte ici, c'est qu'un nouveau thème a été abordé dans le débat, un thème supplémentaire. C'est sur cette idée de supplément qu'insiste l'énonciateur avant de présenter finalement ce thème. Là encore, l'ordre des mots fait sens. Au changement syntaxique, par rapport à l'ordre canonique sujet - prédicat, correspond un changement sémantique avec focalisation sur un élément particulier.

\subsection{Le semi-passif et le passif réciproque}

Cette construction présente des traits adjectivaux, d'où la formulation «semi-passif ». Cependant, il y a bien une forme active correspondante, donc présence d'un agent. Ce type de détermination ne se rencontre qu'avec des verbes dont le procès exprime une comparaison ou une association entre deux éléments, éléments qui apparemment sembleraient pouvoir changer de place sans grande différence sémantique. Ces constructions, qui sont assez productives, ne se retrouvent qu'avec des verbes dont le procès exprime un lien entre deux éléments, que ce soit une comparaison (to compare, to equate...) ou une association (to link, to relate, to associate...). Ces constructions présentent également des traits adjectivaux, bien que l'on puisse retrouver une forme active correspondante. Mais la particularité réside bien dans le fait que ces constructions peuvent s'inverser sans modifier le sens de l'énoncé. C'est pourquoi nous pouvons les 
qualifier de "détermination passive réciproque " ou encore "détermination passive commutative» pour utiliser un vocabulaire plus scientifique. Voici l'un des exemples relevés dans mon corpus :

The possibility that the development and progression of endometriosis is associated with abnormal immune function is the most recent hypothesis for the aetiology of this disease progress. (The Lancet vol. 340, Nov. 21, $1992: 1264$ )

The possibility that abnormal immune function is associated with the development and progression of endometriosis is the most recent hypothesis for the aetiology of this disease progress.

Est-il possible d'appliquer un simple «retournement » et obtenir ainsi un autre énoncé ayant le même sens? Les éléments sont les mêmes dans ces deux énoncés, mais leur distribution est différente, et celle-ci fait sens. Dans le texte de départ, le thème de l'énoncé est the development and progression of endometriosis, alors que dans notre proposition, ce serait abnormal immune function. Or, l'article porte sur l'endométriose, le titre étant «Pathogenesis of endometriosis ». Les conséquences de cette maladie ne doivent donc apparaître qu'en deuxième place, après la présentation du thème principal. Ceci montre bien que, même lorsqu'en apparence l'ordre des mots peut être inversé, sans rien modifier à la structure verbale, ce changement syntaxique entraîne obligatoirement un changement sémantique. Le terme de départ reste le thème de l'énoncé, le sujet du discours, et les autres termes ne viennent qu'en position secondaire par rapport à celuici.

\section{La détermination passive tronquée}

Il existe certains cas où les déterminations simples et complexes peuvent être tronquées, c'est-à-dire qu'un ou plusieurs éléments seront absents. Il ne s'agira bien sûr pas d'un oubli de la part de l'énonciateur, mais d'une volonté de simplifier, de raccourcir son propos en effaçant un terme jugé évident et trop lourd au sein de son énoncé.

\subsection{La détermination passive simple tronquée}

Prenons l'exemple suivant :

As the prevalence of disease falls in children examined, the positive predicative value of cough with fast breathing or chest indrawing will fall, even if sensitivity and specificity remain the same. (The Lancet vol. 338, Dec. 7, 1991 : 1453)

Cet exemple est constitué d'un énoncé pouvant se diviser en trois propositions: une proposition principale encadrée par deux propositions subordonnées. La première de ces subordonnées est As the prevalence of disease falls in children examined. Si cette proposition avait été énoncée seule, nous aurions alors obtenu: The prevalence of disease falls in children who have been examined. Cette relation synthétique permet de supprimer la proposition relative et d'intégrer, voire d'enchâsser, cette détermination passive tronquée dans une autre proposition. Cet enchâssement est centré sur le mot children qui est à la fois $\mathrm{C} 1 \mathrm{du}$ verbe falls et $\mathrm{C} 0$ du verbe examine. Nous en concluons donc que cette diathèse passive tronquée sert à déterminer le mot children.

\section{Concision}

41 Ce type de construction elliptique est relativement fréquent en anglais médical. Il représente 9,5\% des 2115 déterminations passives répertoriées dans notre corpus. Il correspond donc activement au mode de pensée des médecins qui souhaitent apporter un 
maximum d'informations en un minimum de temps, ou d'espace, puisqu'il s'agit là d'énoncés écrits. Cela leur permet une grande économie linguistique dans le rapport entre l'information et l'énoncé. Il ne s'agit donc pas tant d'une réduction du coût mémoriel que d'une réduction du coût énonciatoire qui correspond bien à l'esprit concis et synthétique des médecins. Il faut également noter qu'outre la concision d'un tel énoncé, toutes les marques temporelles aspectuelles et modales disparaissent avec l'effacement du marqueur be. Il ne reste en effet que Co et le participe passé qui, par définition, est une marque invariable renvoyant à l'accompli.

\subsection{La détermination passive complexe tronquée}

Il est à noter que cette troncation, pour autant synthétique qu'elle soit, n'exclut nullement l'instanciation de la place de $\mathrm{CI}$ :

Full and complete compliance is the state in which the patient takes all the prescribed drug for the entire period intended by the prescribing physician. (The Lancet vol. 335, Feb. 3, 1990 : 262)

Là aussi nous pourrions avoir, en rajoutant les éléments effacés, the entire period which was intended by the prescribing physician, ce qui correspond à la relation primitive <physician intend - period>. L'énonciateur "enchâsse" donc cette relation sous forme de détermination passive dans un groupe nominal prépositionnel dont le centre, le thème est l'élément but de la relation primitive. Il synthétise donc ses informations en supprimant des outils grammaticaux vides de sens (relatif which, copule be). Il y a par conséquent concentration syntaxique, mais non sémantique, ce qui n'exclut nullement l'instanciation de la place de l'agent qui est réalisée s'il est besoin d'apporter une détermination supplémentaire.

\subsection{La détermination passive simple tronquée sans $\mathrm{C} 0$}

Il existe enfin un dernier type de troncation que l'on pourrait qualifier d'« extrême » et qui consiste à ne conserver de cette détermination passive que le participe passé. La copule be a disparu, mais également C0 (sans parler de C1 qui de toute façon n'est pas partie intégrante du schéma de base de la détermination passive simple) :

There are three main goals in the treatment of endometriosis - pain relief, resolution of endometriotic deposits, and restoration of fertility, when wanted. (The Lancet vol. 340, Nov. 21, $1992: 1264$ )

Nous constatons bien là que when wanted est la troncation d'une détermination passive qui aurait pu être, si elle avait été complexe, when restoration of fertility is wanted by patients - Cette troncation n'est possible que lorsqu'elle se trouve dans une proposition subordonnée. $\mathrm{C} 0$ et la copule be peuvent disparaître si $\mathrm{C0}$ a déjà été exprimé dans la proposition principale. Nous obtenons ainsi une relation extrêmement serrée entre un élément de la principale et cette subordonnée servant à le préciser et à le déterminer. Cette construction aboutit à une valeur de sens plus générique et concise que si la relation avait été entièrement développée. Prenons enfin un cas encore plus extrême :

Relapse of pain subsequently occured in about one fourth of these, although this figure may not be reliable given the rapid demise of many of the patients. (The Lancet vol. 340, May 28, 1994 : 1008)

Dans cet exemple, nous avons l'utilisation de given qui est alors considéré comme une préposition (Collins $1987: 614$ ). Or cette préposition dont le sens est if what is mentioned is 
taken into account, est la trace d'une détermination passive tronquée. Cette détermination passive développée pourrait se trouver sous la forme if the rapid demise of many of the patients is given, considered or taken into account. Cette concision n'efface nullement la clarté du propos, bien au contraire. Elle affirme la tournure d'esprit des scientifiques que sont les médecins pour qui rigueur, clarté et concision sont indissociables. Cette détermination passive tronquée peut donc aboutir à une conversion: un participe passé devient préposition. Elle peut également, comme nous allons le voir maintenant, donner lieu à une nominalisation.

\subsection{La détermination passive nominalisée}

47 Par détermination passive nominalisée, nous entendons prédicat nominalisé suivi de by et d'un complément d'agent. Ce dernier est la source de la relation prédicative qui apparaitt sous la forme d'une nominalisation. L'agentivité est alors obligatoirement exprimée et relève de ce que nous avons appelé la détermination passive complexe. Cependant, elle est tronquée en ce sens que la copule be disparait, de même que le participe passé - tous deux étant remplacés par ce prédicat nominalisé :

There are many reasons why treatment has not influenced case-fatality rates, most notably poor assessment of the severity of asthma by patients and doctors, and a reluctance to prescribe adequate therapy, especially steroids. (The Lancet vol. 335, Feb. 3, $1990: 262$ )

Poor assessment of the severity of asthma by patients and doctors équivaut approximativement à la détermination passive complexe telle que nous l'avons définie précédemment, soit the severity of asthma was poorly assessed by patients and doctors. Cependant, nous devons nous demander pourquoi le médecin ayant écrit cet article choisit cette construction plutôt que la forme plus développée avec be + participe passé. La solution de facilité consisterait à dire qu'il s'agit là d'une composition lexicale figée dont l'utilisation est rendue obligatoire par certaines contraintes syntaxiques. L'auteur cherche à présenter des raisons à un problème donné, ce qui va entraîner toute une énumération de différentes raisons. Celles-ci vont donc être exprimées sous forme de noms, d'où la nominalisation du prédicat assess the severity of asthma, tout comme nous avons ensuite sur le même plan, le nom a reluctance to prescribe adequate therapy. Or dans cet article, l'énonciateur choisit, grâce à cette nominalisation, de prendre comme thème de son énoncé le prédicat luimême. Le centre du discours n'est pas ici l'agent (médecins et malades) ni même l'objet de l'étude (la gravité de l'asthme), mais l'évaluation de l'objet réalisée par l'agent, c'est-àdire le prédicat. Nous pouvons donc, comme nous l'avons fait, retrouver la relation prédicative sous-jacente; mais avec cette détermination passive nominalisée, elle est présentée de manière tronquée et compacte, ce qui entraîne la disparition de toute marque temporelle, aspectuelle ou modale. Elle renvoie ainsi plus directement à la notion. Il est donc clair que l'utilisation de cette construction correspond à certaines contraintes syntaxiques, mais il faut également dire qu'à celles-ci s'ajoutent certaines contraintes énonciatives, ou pour être plus précis, certaine volonté de la part de l'énonciateur. En effet, toute nominalisation de la détermination passive correspond à une thématisation de ce prédicat. L'énonciateur choisit de présenter non pas $\mathrm{C} 0$ ni même $\mathrm{C} 1$ comme thème de son discours, mais le verbe qui est alors nominalisé, et qui renvoie plus directement à la notion. 


\subsection{Les adjectifs composés}

49 Voyons maintenant les adjectifs composés dont le premier terme est un nom et le deuxième un participe passé. Examinons tout d'abord en quoi il est possible de parler de détermination passive en ce qui les concerne, et en quoi il y a également là troncation.

An effective reduction in the use of salt in the general population has proved to be difficult to achieve but recent evidence strongly suggests that a considerable reduction in sodium intake and in sodium-induced illness can be easily achieved by potassium, magnesium, and lysine enriched alternatives with the same taste and technological properties as common salt. (The Lancet vol. 343, Feb. 26, 1994 : 546)

Nous notons bien que sodium-induced illness présente une relation compacte et synthétique très elliptique sans aucune marque temporelle, aspectuelle et modale. Si nous voulons développer cette relation, l'agentivité apparaît aussitôt : illness induced by sodium. Nous obtenons un cas de détermination passive complexe tronquée, qui est lui-même la troncation de la relation qui est, lorsqu'elle est entièrement développée illness which is induced by sodium. Les deux opérations servant à réaliser cette détermination passive complexe sont donc bien présentes dans cette construction adjectif composé + nom, même si les deux opérateurs be et by disparaissent pour donner une relation très serrée. Cette troncation aboutit donc à une relation compacte qui peut ainsi s'intégrer à toute autre relation, sans alourdir la syntaxe, et sans pour autant s'appauvrir sémantiquement. Nous constatons là encore que concision et clarté vont de pair. Ces adjectifs composés présentent donc de manière très synthétique une relation qui ainsi s'intègre parfaitement à l'énoncé. Ils sont presque toujours utilisés comme épithète de l'élément but de cette relation et, dans ce cas, s'écrivent toujours avec un trait d'union. Ce signe typographique est en quelque sorte le marqueur de la troncation de cette détermination passive complexe. Il est présent dans 108 adjectifs composés sur les 111 que nous avons relevés dans notre corpus. Ces 108 adjectifs composés sont tous utilisés en position d'épithète et donc placés juste avant le nom correspondant à $\mathrm{C} 0$ de la relation développée. Les trois adjectifs composés écrits sans trait d'union sont les trois seuls utilisés comme attribut. Prenons l'un de ces trois cas :

Since $0.01-0.001 \%$ of cells in the patients' peripheral blood were gene marked and the efficiency of marking was $1-5 \%$, between 1 in 100 and 1 in 5000 circulating lymphocytes were probably derived from the infused line. (The Lancet vol. $345 \mathrm{Jan}$. 7, 1995 : 9)

51 L'absence de trait d'union lors de l'utilisation de l'adjectif composé en position d'attribut est justifiée par le fait qu'une seule opération de repérage est tronquée, celle qui est marquée par l'opérateur by. En effet, cells [...] were gene marked correspond à cells [...] were marked by genes. La relation est beaucoup moins synthétique et il n'est pas besoin d'introduire de marque typographique particulière, la détermination passive simple étant, elle, développée normalement avec son marqueur be. L'utilisation du trait d'union est donc tout à fait cohérente en anglais médical en ce qui concerne les adjectifs composés. D'ailleurs, à titre de confirmation, nous avons dans le même texte, cet adjectif composé, mais utilisé cette fois en position d'épithète. Il est alors bien écrit avec un trait d'union.

Gene-marked cells were detected for 10 weeks after the infusion; 10 months after treatment, the patient remains free of complications, including graft-versus-host disease. 


\section{en -ing :}

Transfusion-associated graft-versus-host disease (TA-GVHD) is a rare but hazardous complication caused by transfusion of leucocyte-containing blood. (The Lancet vol. 343, March 26, 1994 : 753)

L'adjectif composé leucocyte-containing ainsi que le nom blood correspondent à la relation primitive < blood - contain - leucocyte > dans laquelle blood est l'élément source et leucocyte l'élément but. Or l'utilisation de l'adjectif composé leucocyte-containing en position d'épithète placé avant le nom blood focalise l'attention sur l'élément but de la relation, à savoir leucocyte. Ceci est d'ailleurs confirmé par le thème de l'article médical d'où est extrait cet exemple. Il s'agit de complications consécutives à la transfusion de sang, et plus exactement de complications causées par les leucocytes se trouvant dans le sang transfusé. Le « thème » de cette relation est donc l'élément but de la relation primitive et nous retrouvons en cela le principe de base de la détermination passive. N'oublions pas, de plus, que dans l'adjectif composé, le premier terme détermine le second et qu'il est en général accentué. Cela montre bien son importance et l'éclairage particulier qui est porté sur ce déterminant, alors que le mot déterminé ne porte qu'un accent faible et qu'il ne sert en quelque sorte que de relateur entre le déterminant de l'adjectif composé et le nom qui suit. Il est très intéressant de remarquer que cet adjectif composé Nom + Verbe en -ing a un sens passif, et que cela est obtenu sans auxiliaire être et sans participe passé. C'est l'orientation du prédicat qui confère à la relation son sens passif. D'autre part, il ne faut pas oublier que -ing peut dans certains cas être considéré comme un marqueur à sens passif :

[...] On the other hand, some words in -ing that might be explained as attributive nouns in -ing are perhaps better regarded as quasi-passive participles (washing tie, cooking apples). Compounds are formed with adverbs and adjectives (wellmeaning, fair-seeming) and with objective nouns (cheese-paring, heart-breaking). ( COD $1964: 625)$.

Nous voyons donc bien que sans les marqueurs « officiels » du passif, l'adjectif composé et le nom leucocyte-containig blood correspondent à l'orientation passive leucocytes are contained in blood. Il s'agit d'ailleurs là d'une opération de localisation et non d'une opération servant à introduire l'agent ou le moyen. Il s'établit donc un parallèle intéressant entre l'adjectif composé de type Nom + Verbe en -ing suivi d'un nom, et la détermination passive complexe. Les marqueurs sont différents, mais l'effet de sens obtenu reste le même. Nous pouvons d'ailleurs construire un autre parallèle tout aussi significatif. Si nous reprenons la relation primitive < blood - contain - leucocyte > nous pouvons respecter l'ordre des éléments et obtenir une détermination active du type blood contains leucocyte. Dans ce cas, le thème du discours, l'élément focalisé sera blood, c'est-àdire l'élément source de la relation primitive. Or, il existe un type d'adjectif composé qui va reprendre cette relation en conservant l'ordre des éléments. Il s'agit de l'adjectif composé de type Nom + Participe Passé. Nous obtiendrons ainsi blood-contained leucocyte. Le paradoxe est que cette expression est la troncation d'une détermination passive complexe leucocytes are contained in blood, mais que l'ordre des mots obtenu correspond à la relation prédicative < blood - contain - leucocyte > Ainsi le nom principal, le thème du discours est bien sûr leucocyte, mais il y a focalisation particulière sur l'élément source blood qui est utilisé comme déterminant dans la détermination adjectivale composée blood-contained. Nous distinguons donc là le jeu subtil de thématisation, voire rethématisation ou surthématisation auquel peuvent aboutir les médecins grâce à 
l'utilisation de ces adjectifs composés - jeu qui se calque sur celui des choix entre diathèse passive ou diathèse active. C'est ainsi à coups d'éclairages particuliers, de projecteurs, de focalisations que des effets de sens spécifiques sont obtenus par les médecins à des fins précises de thématisation.

\subsection{Rôle adjectival du passif statif} la même phrase, ce qui montre le caractère marginal de cette utilisation.

In recent years aid-in-dying initiatives failed in California and Washington state, in large part because their wording allowed for doctors to administer the drugs, raising fears among many voters that the demented and disabled might be put to death without their consent. (The Lancet vol. 344, Nov. 26, 1994 : 1493) partir des verbes to dement et to disable auxquels a été ajoutée la flexion verbale -ed. Si nous recherchons la relation implicite, nous pouvons dire qu'il s'agit d'une détermination passive stative sous-jacente the people who are demented and who are disabled.

Il s'agit donc bien, là encore, d'un phénomène de troncation syntaxique. Il s'apparente au phénomène plus général consistant à employer nominalement un adjectif comme par exemple the rich ou the French. Il renvoie donc à une propriété, reste invariable et est utilisé avec un verbe conjugué au pluriel.

\section{Conclusion}

Je clos cette étude, comme je l'ai commencée, en citant une recommandation aux auteurs afin de les inciter à modérer leur utilisation du passif :

The active is the natural voice, the one in which people usually speak or write and its use is less likely to lead to wordiness and ambiguity. The "passive of modesty ", a device of writers who shun the first-person singular should be avoided. (CBE Style Manual 1978 : 77) 
D'une part, les concepts de « voix naturelle » et de « passif de modestie » me semblent, à la lueur de mon étude, quelque peu erronés. D'autre part, les auteurs de ces conseils enfreignent là encore leurs recommandations en utilisant eux-mêmes un passif! C'est bien la preuve qu'ils méconnaissent le rôle et la fonction de la détermination verbale passive qui la rende indispensable. Ils ne s'intéressent qu'à l'ordre de surface des éléments, par une approche descriptive, sans voir le sens profond implicite que révèle une approche linguistique. Ils ne présentent que la «forme passive» en ignorant la "détermination passive ", qu'il est en fait impossible de moins utiliser. La détermination passive permet une distribution autre des éléments. La traiter uniquement comme un mécanisme de transformation serait réducteur. C'est plutôt un générateur d'énoncés qui correspond à une opération de détermination (voire prédétermination et surdétermination), de thématisation, de focalisation. L'effet obtenu étant souvent une illusion d'objectivité et d'universalité, une concision réelle avec cependant clarté toujours présente. En d'autres termes, l'agent, c'est-à-dire le médecin, est souvent omis pour centrer l'intérêt sur le patient, c'est-à-dire le malade. Ces deux termes prennent effectivement un jour nouveau en anglais médical et semblent tout à fait justifiés !

\section{BIBLIOGRAPHIE}

Albrespit, J. 1994. « Manifestations de la diathèse en anglais contemporain dans le cadre de la théorie des opérations énonciatives ». Thèse de doctorat, Paris 7.

C.B.E. 1978. Style Manual : A Guide for Authors, Editors and publishers in the Biological Sciences. Bethesda, MD : Council of Biology Editors.

Chevalier, J.-C. et alii. 1964. Grammaire Larousse du français contemporain. Paris : Larousse.

Culioli, A. et alii. 1970. Conditions théoriques à propos du traitement formel du langage. $\mathrm{N}^{\circ} 7$ Centre de linguistique quantitative de la Faculté des Sciences de Paris.

Culioli, A. 1975-1976. Transcription du séminaire de D.E.A. D.R.L. Université Paris 7.

Fowler, H.W. \& F.G. (dir.). 1964. The Concise Oxford Dictionary of Current English. Oxford : Oxford University Press.

Palmer, F.R. 1965. A Linguistic Study of the English Verb. Londres : Longman.

Quirk, R. et alii. 1991 [1972]. A Grammar of Contemporary English. Londres : Longman.

Sinclair, J. (dir.). 1987. Collins Cobuild English Language Dictionary. Londres et Glasgow : Collins.

Tortora, G. J. 1992. Principles of Human Anatomy. New York : Harper Collins.

\section{RÉSUMÉS}

À partir d'exemples caractéristiques issus d'un corpus de 2115 structures passives tirées de la revue britannique The Lancet, j'ai réalisé une classification originale de ces diathèses passives en 
trois grandes catégories, à savoir la détermination passive simple, la détermination passive complexe et la détermination passive tronquée. Après avoir précisé en quoi ces opérations de détermination correspondaient à une stratégie énonciative particulière - qu'elle soit réfléchie ou spontanée - mise en œuvre par les médecins, j’ai analysé quelques formes extrêmes, ou déterminations particulières, notamment celles présentées sous la forme d'adjectifs composés.

Thanks to 2,115 passive structures collected in the British journal The Lancet, I designed an original classification of these passive specifications in three large categories, through an utterer-centered approach to linguistic grammar. After showing how these specification operations lead to an original enunciative strategy - whether it be carefully thought over or spontaneous - I analysed a few extreme forms (or particular specifications) especially those in the form of compound adjectives.

\section{INDEX}

Mots-clés : anglais médical, concision, détermination passive, objectivité, pré-détermination, stratégie énonciative, thématisation

Keywords : conciseness, enunciative strategy, medical English, objectivity, passive specification, pre-specification, topicalisation

\section{AUTEUR}

\section{DIDIER CARNET}

